\title{
The Effect of Video Feedback of Social Comparison on Activity of Mirror Neurons and Golf putt Learning in Young Girls
}

\author{
Narjes Yavari ${ }^{a *}$, Masoumeh Shojaei ${ }^{b}$ Afkham Daneshfar $^{c}$ \\ ${ }^{a}$ Master Degree in Motor Behavior, Faculty of Sport Sciences, Alzahra University, Tehran, Iran \\ ${ }^{\mathrm{b}}$ Associate Professor, Department of Motor Behavior, Faculty of Sport Sciences, Alzahra University, Tehran, Iran \\ ${ }^{\mathrm{c}}$ Associate Professor, Department of Motor Behavior, Faculty of Sport Sciences, Alzahra University, Tehran, Iran
}

\begin{tabular}{l}
\hline Keywords \\
\hline Social Comparison Feedback \\
Video Feedback \\
Normative Feedback \\
Golf Putt \\
Mirror Neurons
\end{tabular}

Narjes Yavari

Email: n.yavari67@yahoo.com

\section{Introduction}

One kind of feedback is social or normative comparison feedback. This feedback includes information on comparing an individual with others or social comparisons that can affect learner motivation, performance and learning. In this kind of feedback, learner will be aware of his score and performance of peer group (Hutchinson et al., 2008). Lewthwaite and Wulf (2010) examined the effects of social comparisons feedback on learning students' motor skills equilibrium assignment.
Participants were divided into three groups: positive, negative and control. After two days practice, which each day included seven attempts in 90 seconds, they received feedback after each attempt. There were provided real feedback for the control group, while the positive and the negative groups received $20 \%$ less and 20\% more than individual's score. It was concluded that individuals in positive and the negative groups had better learning than the control group, but it was more effective in the positive feedback group. In 
general, positive social comparison feedback has a facilitating effect on motor learning (Lewthwaite \& Wulf 2010).

Another research was conducted by Rashidi (2013) in field of social comparison feedback to compare the effect of positive and negative social feedback on performance accuracy, electromyography of shoulder and arm muscles and kinematics of elbow in Dart throw. The research results showed that positive feedback effect is not correlated with performance accuracy of physical arousal, and improves performance by improving the elbow angle while releasing the dart. These findings indicate that motivational factors affect performance and motor learning (Rashidi 2013).

The conducted studies on mirror neurons also suggest that, in addition to the power of imitation, these neurons play a prominent role to stimulate emotions; in other words, activity of these neurons is influenced by motivational factors. For example, a research using the Transcranial Magnetic Stimulation (TMS) showed that participants who observed positive or negative images indicated increasing cortical excitability in comparison to those who viewed neutral images (Hajcak., et al 2007). Another study, using a TMS pattern concluded that negative stimulation (relative to positive stimulation) facilitates spinal cord excitability in response to observation of movement of the hand (Enticott., et al 2012). Hill et al. (2013) showed that activity of neuronal system was increased in response to negative and positive stimulation toward neutral stimulation (Hill., et al 2013).

In the above-mentioned research, we are faced with a kind of contradiction that has been seen about the mechanism of social comparison feedback. Some studies have indicated that motivational effect of positive normative feedback has improved performance and, in some cases, it has been attributed to other factors. However, in studies related to mirror neurons, it was also observed that negative stimulation activates these neurons further. Because social comparison feedback and activity of mirror neurons are both associated with stimulating individual's emotions, and as each of them suggests a different type of stimulation of emotion to improve performance, this contrast can be seen in the effectiveness of individual's sense of feeling on learning. Also, due to lack of adequate research on mirror neurons in the field of sports skills, the question posed here is whether social comparison feedback (positive, negative and real) can affect the magnitude or activity of mirror neurons in positive, negative and neutral feelings in golf putt differently. So, here it is necessary to address the role of social comparison video feedback and whether this feedback can be affected by activation of mirror neurons by neurological examination.

\section{Method}

A pretest-posttest design of random groups was applied to conduct this quasi-experimental research. The research statistical population consisted of all non-athlete female students of Al- 
Zahra University who were studying in the second semester of 2013-2014 with age range of 20-30 years old. Participants, in addition to being totally healthy and with a superior dominant left hemisphere, should not have any previous experience on golf putt. Since, according to the conducted study by Karimi (2013), self-efficacy may affect the type of perception of social comparison feedback in individuals; for controlling this psychological factor, based on the self-efficacy questionnaire, those participants with very high self-efficacy (80-100) or very low self-efficacy (020) were deleted. Finally, 18 people were selected as the sample.

After a brief explanation of how to perform the tests and duration of the research, volunteer participants were randomly selected, and written consent was obtained. The mean age of the participants in the study was $24.83 \pm 2.09$. They were randomly classified into three groups: social positive comparison video feedback, negative social comparison feedback group and real video feedback.

Participants' duty was to hit the golf ball and guide the ball towards the goal. The target was a circle with radius of $5 \mathrm{~cm}$, located at a distance of 4 meters from the participants. There were designed 14 concentric circles with radii $10,15,20,25 \ldots 75 \mathrm{~cm}$ around the target and the circles were labeled with their own points. These instruments were used as a measure for the accuracy of launchers. If the ball is on area A (the goal is the same hole in golf sport), there will be considered 150 points; and placing the ball on other areas show the following points: B (140), C (130), D (120) and.... O (10), and if it is outside of the zone, it will be considered 0 points (Cross et al., 2006).

The used tool in the present research includes biofeedback system (Procomp-2), which is a tool equipped with a computer system that is used to implement a nerve feedback training method. This tool uses devices that are connected to the body (electrodes) to provide information about some of biological functions of individual's body. Electroencephalogram sensor (EEG) or electroencephalography is the recording of brain wave signals available from cerebellum in digital or paper form. Through electrodes attached to the skull, EEG device records activities of brain neurons and shows them in strips of different shapes. It was used a computer with the installed application for recording information related to electrical activity of the brain and electrical activity of muscles; and also used another computer to display video feedback. However, we used a professional camera (BetaCam) to record videos related to the person's performances to give feedback; And also used tools for golf sport (club putter, ball) and carpet, as well as side-by-side questionnaire. The questionnaire was self-report and included 16 items (4 items for each of the following four subscales: handedness, footedness, eyedness and earedness). The questionnaire score is between -16 and +16 . Based on the research purpose, participants with scores from -10 to -16 were ranked in the dominant right hemisphere group, and those with scores from +10 to +16 were 
classified in the dominant left hemisphere group (Coren 1993). The questionnaire content validity was approved by five experts and its internal consistency was reported by Cronbach's alpha (0.89). The questionnaire simultaneous validity was evaluated in subscale of superior hand score with Annette's handover questionnaire (1970) (Annett 1970) and in footedness subscale was evaluated using Waterloo Footedness Questionnaire (1998), which showed significant correlation coefficient. The researcher-made selfefficacy questionnaire using Bandura's (2006) guidelines was provided, which is graded in range of zero (unreliable) to 100 (complete confidence) and in 10 units. The questionnaire construct validity was obtained based on intergroup variations (two groups of 18 skilled and beginner people) due to exercise skill of golf putt technique using significant independent $t$ statistical methods $(p=0.0006, t=4.566)$.

Before conducting main stage of the experiment, a preliminary study was conducted (a pilot study) on 10 non-sample members to obtain information on stages of testing and identifying possible problems. We were selected 18 people from those with a superior dominant left hemisphere with a moderate self-efficacy score, after filling Corn superiority questionnaire and self-efficacy questionnaire. After selecting the sample and filling out the consent form, people were given explanation about the motion, and the participants were classified in three groups: positive social comparison video feedback, negative social comparison video and real video feedback (each group contained of six individuals). All groups participated in three sessions (pretest, acquisition and retention) for three consecutive days (Rodriguez et al., 2013). At all sessions, the related electrodes to record electrical activity of the brain were installed on the $\mathrm{Cz}$ spot (the region showing activity of mirror neurons) on the head. A camera is located perpendicular to the trajectory path that records all his movements from the beginning of swing of the ball until the end of ball movement. By recording the movements, these videos were used to give feedback. The participant observed the goal before moving, but when swing, to prevent visual feedback, a paper jam that stood next to his face was used to prevent seeing the target.

At the beginning of the test, the examiner gave the required educations to the subjects. Educations on how to get the club in hand, how to put the ball back and then putt swing, which included kicking the club, swing and moving the club; putt swing was only shown to the participants only once. To control novelty of the participants, they made several attempts in form of a test. Subsequently, the subjects performed six attempts at the pretest session, and there were recorded information regarding activity of the mirror neurons and accuracy of swings in three positions: basic (person sitting on the chair and looking at a black plate exactly on his face), execution (during execution of the total six attempts, there was conducted recording) and observation. In terms of observation, all participants' data were recorded at time of receiving real video feedback from their 
own efforts. On the next day (acquisition period), individuals performed 60 attempts, which were divided into 10 categories of effort (each with 6 attempts). There were 5 minutes of rest after each attempt. At the end of effort groups and at rest time, intervals received appropriate feedback from the same group according to the groups in which they were placed. In feedback groups, in the last attempt of acquisition step, in three basic, observation and execution conditions, there were recorded amount of electrical activity of the brain and accuracy of swings. In positive social comparison video feedback, after the individual made his own attempt, there were provided firstly the film (showing total duration of journey from start to finish, as well as the goal) of weak attempt of the other three persons (normative feedback) that was pre-made, and then a movie was shown from other three best-of-film, as unrealistic feedback but as examples of how people performed in previous experiments. In negative social comparison video feedback, after the individual performed his attempt, there were provided firstly the film (showing total duration of execution from start to finish, as well as the goal) of better attempt of the other three persons (normative feedback) that was pre-made, and then a movie was shown from other three worst-of-films, as unrealistic feedback but as examples of how people performed in previous experiments, and then in movie 3 , the weaker one's own effort was shown to him. In the real feedback group, after the individual made his own effort, there was shown film shot of all his six own efforts as real feedback. The retention session was also held 24 hours after the acquisition session, which had totally six attempts. At this stage, all information about brain activity and swing accuracy were recorded in the following three conditions: basic, observation (people in all groups received only real feedback) and execution (total 6 attempts), which was observed in observation conditions (similar to pretest conditions) and information of all groups at the time of actual video feedback from one's own efforts.

In order to analyze the data, there were used descriptive statistics of swing accuracy, Mu rhythm suppression (logarithm of ratio of power when observing and executing to the base position power), in order to control variability in absolute $\mathrm{Mu}$ rhythm suppression as a result of individual differences such as skull thickness and electrode resistance. Since the ratio data are not inherently natural, the data were changed to lo before analyzing). Fourier analysis was used to convert waves into numbers and figures. To analyze the waves related to activity of mirror neurons, oneway ANOVA was used for comparing pretests and retention test for measuring accuracy of swings and the degree of Mu rhythm suppression; combination variance analysis $(10 * 3)$ with the repeated measurement in the last factor (effort category) was used to compare precision of swings in the acquisition sessions; also, combination variance analysis $2 * 3 * 3$ with repeating measurement of the last two factors (test status) was used for analyzing $\mathrm{Mu}$ rhythm suppression data; the dependent t test was used for paired comparisons of three-factor interactions. There was considered significant level 
of 0.05. SPSS software Version 19 was used for data analysis.
Table 1 shows descriptive statistics of the accuracy of golf putt in different groups. Figure 1 also shows the average accuracy of golf putt in different groups.

\section{Results}

Table 1. Descriptive statistics of accuracy execution of participants in the investigated groups at different stages.

\begin{tabular}{|c|c|c|c|c|c|c|c|}
\hline \multicolumn{2}{|c|}{ Time steps } & \multicolumn{2}{|c|}{$\begin{array}{c}\text { Positive social comparison } \\
\text { video feedback }\end{array}$} & \multicolumn{2}{|c|}{$\begin{array}{l}\text { Negative social comparison } \\
\text { video feedback }\end{array}$} & \multicolumn{2}{|c|}{ Real video feedback } \\
\hline Statistic & & Average & $\begin{array}{l}\text { Standard } \\
\text { deviation }\end{array}$ & Average & $\begin{array}{l}\text { Standard } \\
\text { deviation }\end{array}$ & Average & $\begin{array}{l}\text { Standard } \\
\text { deviation }\end{array}$ \\
\hline Pretest & & 95.50 & 35.31 & 101 & 22.14 & 88 & 33.26 \\
\hline \multirow{10}{*}{ Acquisition } & Attempt 1 & 89 & 23.24 & 84 & 28.77 & 100.50 & 24.76 \\
\hline & Attempt 2 & 77.50 & 28.30 & 81.33 & 32.81 & 82.17 & 37.33 \\
\hline & Attempt 3 & 77.17 & 29.73 & 96.67 & 28.92 & 100.17 & 20.14 \\
\hline & Attempt 4 & 109 & 28.51 & 111.67 & 15.49 & 100.67 & 25.30 \\
\hline & Attempt 5 & 115.83 & 21.66 & 115.50 & 22.79 & 98.83 & 15.08 \\
\hline & Attempt 6 & 98.50 & 17.76 & 97.67 & 18.77 & 107 & 31.26 \\
\hline & Attempt 7 & 101.33 & 37.32 & 105.67 & 30 & 109.17 & 43.31 \\
\hline & Attempt 8 & 119.67 & 15.77 & 109.17 & 19.99 & 109.67 & 15.96 \\
\hline & Attempt 9 & 113.83 & 26.71 & 94 & 26.89 & 93.17 & 35.69 \\
\hline & Attempt 10 & 92.50 & 12.98 & 114 & 31.24 & 101.50 & 20.17 \\
\hline Retention & & 77.50 & 29.34 & 105 & 25.38 & 104.33 & 14.72 \\
\hline
\end{tabular}

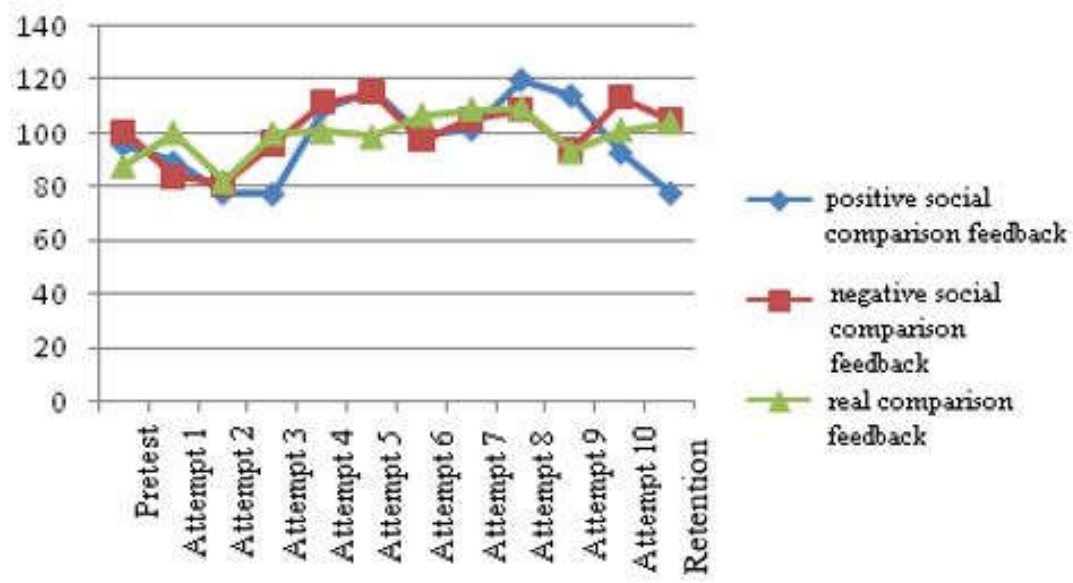

Fig. 1. The average score of execution accuracy of the examined groups at different stages.

Table 2 shows descriptive statistics on activity of mirror neurons in brain (Mu rhythm suppression) in different groups. Figure 2 also shows the average activity of brain neurons in brain (Mu rhythm suppression) in different groups at execution time, and Figure 3 shows the mean of activity of mirror neurons in brain (Mu rhythm suppression) in different groups at the time of observation. 
Table 2. Mean and standard deviation of Mu rhythm suppression at different stages and the examined groups.

\begin{tabular}{|c|c|c|c|c|c|c|c|}
\hline \multirow[b]{2}{*}{ Group } & \multirow[b]{2}{*}{ level } & \multicolumn{6}{|c|}{ Mu rhythm suppression } \\
\hline & & $\begin{array}{l}\text { Average } \\
\text { of pretest } \\
\text { stage }\end{array}$ & $\begin{array}{l}\text { SD of } \\
\text { pretest } \\
\text { stage }\end{array}$ & $\begin{array}{l}\text { Average of } \\
\text { acquisition } \\
\text { stage }\end{array}$ & $\begin{array}{l}\text { SD of } \\
\text { acquisition } \\
\text { stage }\end{array}$ & $\begin{array}{l}\text { Average } \\
\text { of } \\
\text { retention } \\
\text { period }\end{array}$ & $\begin{array}{l}\text { SD of the } \\
\text { retention } \\
\text { period }\end{array}$ \\
\hline \multirow{2}{*}{$\begin{array}{l}\text { Positive social } \\
\text { comparison } \\
\text { feedback }\end{array}$} & execution & 0.1598 & 0.2072 & -0.0049 & 03496 & 0.0192 & 0.2701 \\
\hline & Observation & 0.0572 & 0.1681 & 0.1663 & 0.3445 & 0.1170 & 0.3938 \\
\hline \multirow{2}{*}{$\begin{array}{l}\text { Negative } \\
\text { social } \\
\text { comparison } \\
\text { feedback } \\
\end{array}$} & execution & 0.2001 & 0.1482 & 0.0552 & 0.2346 & -0.1672 & 0.4753 \\
\hline & Observation & -0.0643 & 0.2130 & -0.0035 & 0.2401 & -0.1749 & 0.4262 \\
\hline \multirow{2}{*}{$\begin{array}{l}\text { Real } \\
\text { comparison } \\
\text { feedback }\end{array}$} & execution & 0.0903 & 0.0896 & 0.0554 & 0.2480 & 0.3019 & 0.2293 \\
\hline & Observation & 0.0056 & 0.1475 & 0.0697 & 0.3395 & 0.0781 & 0.6602 \\
\hline
\end{tabular}

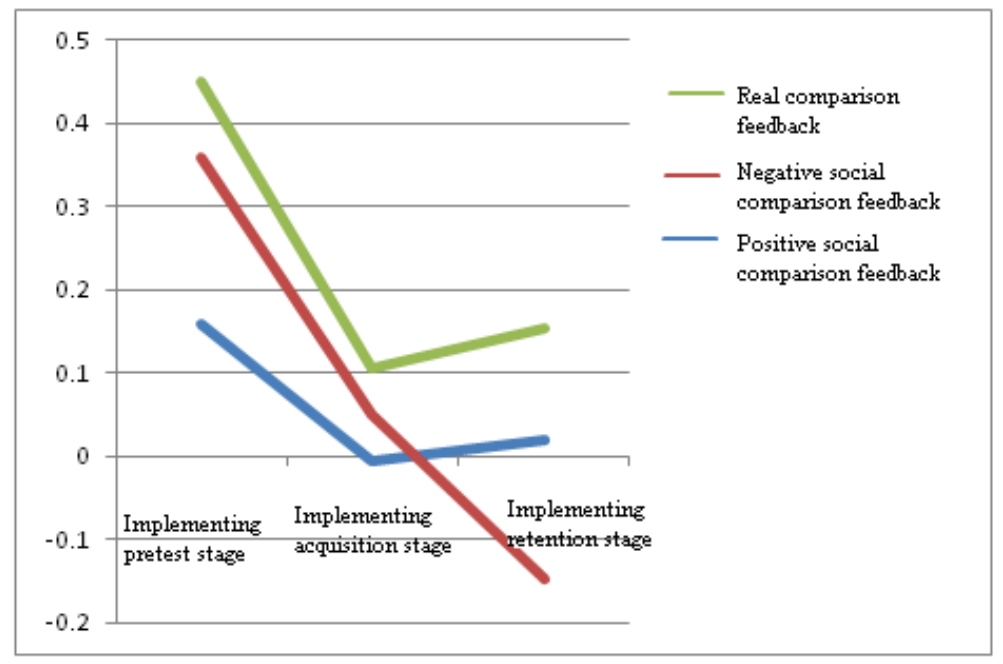

Fig. 2. Average of Mu rhythm suppression at execution time in different groups and sessions.

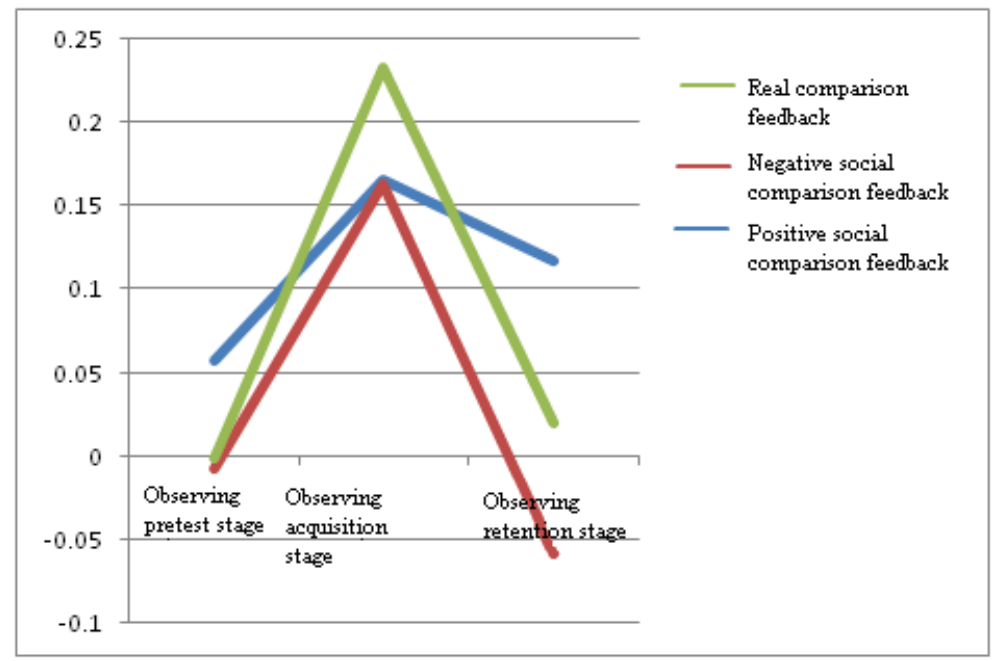

Fig. 3. Average of Mu rhythm suppression at observation time in different groups and sessions.

With the assurance of normal distribution of scores and homogeneity of variances, there were used variance analysis (group 3) tests in 10 (category effort) by repeating measurement of the 
experimental group to evaluate the effect of positive social comparison video feedback, negative social comparison video feedback and real video feedback on accuracy of swing on the acquisition stage. The results showed that the main effect of effort group was significant $\left(\eta^{2}=0.150\right.$; $\mathrm{P}=0.038 ; \mathrm{F}=2.645 ; \mathrm{df}=9$ ). According to the results of Bonferroni test, the accuracy of the swings was only significant between attempt 2 and $8(\mathrm{P}=$ 0.040) and other groups did not have a significant difference $(\mathrm{P}>0.05)$. On the other hand, the effect of the main factor of the group $\left(\eta^{2}=0.005, P=\right.$ 0.965, $\mathrm{F}=0.36, \mathrm{df}=2$ ) and interaction effect between agents and groups $\left(\eta^{2}=0.083, P=0.722\right.$, $\mathrm{F}=0.675, \mathrm{df}=18$ ) was not significant. There is no significant difference between the different groups in terms of operation accuracy. However, in all groups, individuals in both groups showed significant differences in terms of execution accuracy. The results of one-way ANOVA, in comparing pretests, showed that there is no significant difference between the three groups at the beginning $\left(p=0.767, F_{(15.2)}=0.270, d f=2\right)$. One-way ANOVA was used to compare the effect positive comparison video feedback, negative social comparison feedback group and real video feedback on accuracy execution of swing in retention period. According to the results in retention phase, there are also no significant differences between the three feedback groups $(\mathrm{P}=$ $\left.0.109, \mathrm{~F}_{(15.2)}=2.573, \mathrm{df}=2\right)$.

Tables 3 and 4 show an inter-group comparison of pretests using one-way ANOVA, which there was no a significant difference between $\mathrm{Cz} \mathrm{Mu}$ rhythm suppression for observation and execution status; therefore, for analyzing data on $\mathrm{Mu}$ rhythm suppression, there was used analysis of variance 3 (group) in 3 (tests) in 2 (condition) with repeat measurement of two last factors.

Table 3. One-way ANOVA results to compare the mean of Mu rhythm suppression in pretests of the execution status.

\begin{tabular}{|c|c|c|c|c|c|}
\hline Group & Average & SD & Df & Sig. & $\mathrm{F}$ \\
\hline $\begin{array}{l}\text { Positive social comparison video } \\
\text { feedback }\end{array}$ & 0.7598 & 0.2072 & \multirow{3}{*}{2} & \multirow{3}{*}{0.484} & \multirow{3}{*}{0.762} \\
\hline $\begin{array}{c}\text { Negative social comparison feedback } \\
\text { group }\end{array}$ & 0.2001 & 0.1482 & & & \\
\hline Real video feedback & 0.0903 & 0.0896 & & & \\
\hline
\end{tabular}

Table 4. One-way ANOVA results to compare the mean of Mu rhythm suppression in pretests of the observation status.

\begin{tabular}{|c|c|c|c|c|c|}
\hline Group & Average & $\mathrm{SD}$ & $\mathrm{df}$ & Sig. & $\mathrm{F}$ \\
\hline $\begin{array}{c}\text { Positive social comparison video } \\
\text { feedback }\end{array}$ & 0.1572 & 0.1681 & \multirow{3}{*}{2} & \multirow{3}{*}{0.512} & \multirow{3}{*}{0.701} \\
\hline $\begin{array}{l}\text { Negative social comparison feedback } \\
\text { group }\end{array}$ & -0.0643 & 0.2130 & & & \\
\hline Real video feedback & 0.0056 & 0.1474 & & & \\
\hline
\end{tabular}


The results of analysis of variance 3 (groups) in 3 (tests) in 2 (situation) with repeating evaluation of the last two $\mathrm{Mu}$ rhythm suppression factors are shown in Table 5. As it is seen, only the main effect of situation was significant $(\mathrm{P}=0.003)$. According to the results, the $\mathrm{Mu}$ rhythm suppression in observation situation (-0.12) was significantly less than execution situation (0.093).

Table 5. Results of analyzing variance of 3 factors for the Mu rhythm suppression variable.

\begin{tabular}{llcrrr}
\hline Source of change & $\mathrm{F}$ & $\begin{array}{c}\mathrm{df} \\
\text { assumption }\end{array}$ & $\begin{array}{c}\mathrm{df} \\
\text { error }\end{array}$ & $\mathrm{p}$ & $\eta^{2}$ \\
\hline Group & 1.125 & 2 & 15 & 0.350 & 0.130 \\
\hline Situation & 12.488 & 1 & 15 & 0.003 & 0.454 \\
\hline Test & 0.005 & 2 & 30 & 0.995 & 0.003 \\
\hline Situation * group & 0.406 & 2 & 15 & 0.673 & 0.051 \\
\hline Test * group & 1.241 & 4 & 30 & 0.315 & 0.142 \\
\hline Situation * test & 0.826 & 2 & 30 & 0.447 & 0.052 \\
\hline Situation * test * group & 1.089 & 4 & 30 & 0.380 & 0.127 \\
\hline
\end{tabular}

\section{Discussion and Conclusion}

The present research aimed to investigate the effect of positive, negative and real positive, negative social comparison video feedback on the activity of mirror neurons in the brain. In the present research, we used a different way from previous research for social comparative (normative) comparison feedback. There are two main differences with other researches in this area: in the conducted studies, feedback was expressed quantitatively to individuals, but in this research, the feedback was given qualitatively for individuals through viewing film of the same age group. Another difference that can be mentioned is that in the previous studies, people who were in the feedback group received more feedback in comparison to the control group participants or the actual feedback group; they received normative feedback, in addition to real feedback. However, as stated, here, the number of feedbacks in all groups is controlled.
The obtained results showed that there was no significant difference between the effect of positive social comparison video feedback, negative social comparison feedback and real video feedback on the accuracy of swing on the retention and acquisition stage, but in all groups, individuals showed significant differences in terms of accuracy in attempts 2 and 8. One of points that can be attributed to these results is that perhaps installation of electrodes overhead and the involved muscle in the final tests limited persons to run swings. The effect of psychological empirical conditions and camera during their performance can also increase the arousal and influence on performance. For these reasons, they have not been able to perform their best performance in category of final attempts, but it cannot be said that the person did not learn the skill. In other words, it may have artificial effects on execution that has covered the effects on learning. In the present study, there was no choice but to control the disparate variables and increase the internal validity. 
The results on lack of difference on accuracy of swings in the three groups confirm Panahi research (2013) that there is no significant difference between the effects of different types of feedback on acquisition (Panahi Borujeni 2012). Of course, Panahi research is consistent with the results of non-difference between the two groups of positive and negative normative feedback. However, the research results are in contradiction with the conducted studies by Lewthwaite and Wulf (2010), Wulf, Chiviacowsky and Lewthwaite (2010), Lucana, Chiviacowsky, Wulf and Lewthwaite (2012), Stoate, Wulf and Lewthwaite (2012), which all of them confirm the effect of normative feedback. As noted earlier, the reason for this difference is probably the provided quantitative amount of normative feedback in the studies and expression of individuals' accuracy verbally, which has improved performance of individuals. Perhaps it's likely that comparing numbers quantitatively creates more motivational effects than comparing two films. Also, the difference in number of the provided feedback in different groups can be one of the contradictory reasons. It is necessary to mention that in previous research, which normative feedback was better than real feedback, it was probably due to large amount of the provided information. Also, these results are in contrast to guidance view. According to this viewpoint, feedback is so important after making poorer attempts or bigger errors to correct motions, and helps learners achieve better learning. Individuals in negative social comparison video feedback, given that they received feedback to their poor efforts, did not differ in comparison with other groups. The reason for the contradiction can be attributed to differences in feedback type (quantitative or qualitative), lack of control of frequency of feedback, as well as inconsistent listening feedback with the observed visual feedback.

Other findings of the research show that in the three groups, comparative-social video feedback has a significant difference in activity of mirror neurons, in terms of observation and execution. In other words, activity of mirror neurons in terms of observation was greater than execution conditions. This type of feedback, which provided to the participants in the same film, in addition to allow individuals to view their performance, would probably have made more neurons active at the time of observation than the execution time. These results are consistent with previous studies such as Vogt et al (2007), Aglioti et al (2008), Cross et al (2009) and Kim et al (2011), which showed increasing activity of these neurons in observation time. The results confirm activation of mirror neurons during observation relative to the execution time.

Another interesting finding that can be extracted from the research is that there was no significant difference between the three groups in activity rate of mirror neurons in execution time. Also, there was no significant difference between the three groups in activity of mirror neurons during observation time. That is, when the participants observe their performance film, there was no difference in viewing the video of their 
weak and strong efforts, or even real efforts, and in any case; in any case, these neurons are activated despite the film observation. If observation of these films caused positive or negative emotions in one person and could not affect activity of mirror neurons, it can be argued that there is required more time to stimulate and activate the neurons. Perhaps it's just because stimulation of emotion is important, rather than stimulating it positively, negatively or neutrally. Perhaps the normative feedback films have not created positive or negative feelings, and maybe due to the lack of homework competition, the positive and negative feedback has no positive and negative emotions that could make a difference between neuronal activities. This conclusion is opposed to the conducted research by Hill et al (2013), Enticott et al (2012) and Hajcak et al (2007). The researchers have found the impact of positive and negative images more than neutral images. The reason for this discrepancy is to show different types of images and using different instruments, as well as measuring just one area of the brain to examine activity of the neurons, which provides less accurate results. The images that were shown to people in this study were such that created a negative and positive feeling in one person, and they were not necessarily images that exactly relate to one's own performance. Perhaps, if the images show the person performance, this level could not stimulate his feelings, and then there was no difference in activation of the neurons in different conditions. With regards of results, studying the origin of eliciting mirror neurons is suggested.
There is also evidence that there is a relationship between emotional processes and mirror neuron system. Numerous studies of neuroimaging have also shown an important role in visualization of emotional state of others. Hill and colleagues (2013) used stimulation TMS to measure activity of mirror neurons. They studied 20 participants who observed three distinct attempts including TMS stimulation conditions together with positive, neutral and negative images, motion picture of moving a cup with the right and left hands in steady and dynamic method. The results showed that in response to positive and negative stimulation, activity of the mirror neuron system was increased to neutral stimulation. They said that observing negative images would create a negative state for participants, while observing positive images does not make any changes in their state (Hill et al., 2013). One of the other points in the conducted study by Hill and colleagues is that, as observation of action is a motivator of the mirror neuron system, it may be neutral stimulation as a suppressor of these neurons; but given the fact that our results did not differ significantly between different groups, we can say that there is still room for questioning and research in this regard. The conducted study by Cross (2006) showed that longterm physical exercises increase activity of these neurons (Cross et al., 2006). Here the exercise took place in a short time. It is likely that the longer execution can cause observing the required differences in different groups. Studying effect of long-term exercises on activity of mirror neurons in the brain is suggested. 


\section{References}

1. Aglioti, S.M., Cesari, P., Romani, M, \& Urgesi, C. (2008). Action anticipation and motor resonance in elite basketball players. Nature Neuroscience. 11: 1109-16.

2. Annett, M. (1970). The classification of hand preference by association analysis. The British Journal of Psychology. 61 (3): 303-321.

3. Badami, R., Vaezmousavi, M., Wulf, G., \& Namazizade, M. (2012). Feedback about more accurate versus less accurate trials: Differential effects on self-confidence and activation. Research Quarterly for Exercise and Sport. 83, (2): 1-8.

4. Coren, S. (1993). The lateral preference inventory for measurement of handedness, footedness, eyedness and earedness: Norms for young adults. Bulletin of the Psychonomic Society. 31 (1): 1-3.

5. Cross, E.S., Hamilton, A.F., \& Grafton, S.T. (2006). Building a motor situation de novo: observation of dance by dancers. Neuro image, 31, 1257-1267.

6. Elias, L.J., Bryden, M.P., \& Butman-Fieming, M.B. (1998). Footedness is a better predictor than is handedness of emotional lateralization. Neuroasychologia, 36 (1), 37-43.

7. Enticott, P.G., Harrison, B.A., Arnold, S.L., Nibaldi, K., Segrave, R.A., Fitzgibbon, B.M., Kennedy, H.A., Lau, K., \& Fitzgerald, P.B. (2012). Emotional valence modulates putative mirror neuron activity. Neuroscience Letters. 508: 56-59.

8. Hajcak, G., Molnar, C., George, M.S., Bolger, K., Koola, J., \& Nahas, Z. (2007). Emotion facilitates action: A transcranial magnetic stimulation study of motor cortex excitability during picture viewing. Psychophysiology. 44: 91-97.

9. Hill, A.T., Fitzgibbona, B.M., Arnolda, S.L., Rinehart, N.J., Fitzgeralda, P.B., \& Enticotta, P.G. (2013). Modulation of putative mirror neuron activity by both positively and negatively valenced affective stimuli: A TMS study. Behavioral Brain Research. 249: 116-123.

10. Hutchinson, J.C., Sherman, T., \& Martinovic, N. (2008). The effect of manipulated self-efficacy on perceived and sustained effort. Applied Sport Psychology. 20: 457-472.

11. Kim, Y.T., Seo, J.H., Song, H.J., Yoo, D.S., Lee, H.J., Lee, J., Kwon, E., Kim, J.G., \& Chang, Y. (2011). "Neural correlates related to action observation in expert archers". Behavioural Brain Research, 223,342-347.

12. Lewthwaite, R., \& Wulf, G. (2010). "Social-comparative feedback affects motor skill learning". The Quarterly Journal of Experimental Psychology, 63(4)738-749.

13. Lucana, T.G., Chiviacowsky, S.A., Wulf, G., \& Lewthwaite, R. (2012). "Positive social-comparative feedback enhances motor learning in children". Psychology of Sport and Exercise, 13, 849-853.
14. Magil, R. A. (2011). Motor learning and control ( $9^{\text {th }}$ Ed.). NewYork: McGraw-Hill.

15. Panahi Borujeni, E. (2012). The effect of feedback on successful, normative and self-control efforts on motivation, execution and learning of badminton service skills in 10-12 years old girls. Master's Thesis. AlZahra University.

16. Prinz, W. (1997). "Perception and action planning". European Journal of Cognitive Psychology, 9, 129-154.

17. Rashidi, F. (2013). The effect of normative feedback on accuracy of performance, electromyography and kinematics of dart throw. Master's Thesis. AlZahra University.

18. Rizzolatti, G., \& Arbib, M.A. (1998). "language within our grasp". Trends in neurosciences, 21,188-194.

19. Rizzolatti, G., \& Craighero, L. (2004). "The mirrorneuron system". Annual Review of Neuroscience.27, 169-192.

20. Rodriguez, A.L., Alonso, V.L., \& del-Olmo, M. F. (2013). Mirror neuron system and observational learning: Behavioral and neurophysiological evidence. Behavioural Brain Research, 248, 104-113.

21. Stoate, I., Wulf, G. ,\& Lewthwaite, R. (2012). "Enhanced expectancies improve movement efficiency in runners". Journal of Sport Sciences, 1-9.

22. Vogt, S., Buccino, G., Wohlschlaeger, A.M., Canessa, N., Shah, N.J., Zilles, K., Eickhoff, S.B., Freund, H.J., Rizzolatti, G., \& Fink, G.R. (2007). "Prefrontal involvement in imitation learning of hand actions: effects of practice and expertise". Neuroimage, 37, 1371-1383.

23. Wulf, G., Chivacowsky, S., \& Lewthwaite, R. (2010). "Normative feedback effects on learning a timing task". Research Quarterly for Exercise and Sport, 81(4), 425431. 